\title{
Collagen/Hydroxyapatite Composite Supports for Bone Tissue Engineering
}

\author{
Roxana Tiplea $^{1}$, Madalina Georgiana Albu-Kaya ${ }^{2}$, Maria Sonmez ${ }^{2}$, Mihaela Ghica ${ }^{3}$, Denisa Ficai ${ }^{1}$, Anton \\ Ficai $^{1}$, Ioana Lavinia Ardelean', Gudovan Dragos ${ }^{1}$, Ecaterina Andronescu ${ }^{1}$ \\ ${ }^{1}$ Department of Science and Engineering of Oxide Materials and Nanomaterials, Faculty of Applied Chemistry and \\ Materials Science, University Politehnica of Bucharest \\ Polizu Street no 1-7, 011061, Bucharest, Romania \\ denisa.ficai@upb.ro \\ ${ }^{2}$ National Research, Development Institute for Textiles and Leather-division: Leather and Footwear Research \\ Institute \\ Bucharest, Romania \\ ${ }^{3}$ Faculty of Pharmacy, University of Medicine, Pharmacy "Carol Davila" \\ Bucharest, Romania
}

\section{Extended Abstract}

Controlled release systems are particularly used in medical applications due to reduced systemic toxicity [1, 2]. The advantage of COLL/HA/drug system comes from the regenerative role induced by the support materials (COLL/HA) as well as the curative activity induced by the delivery of the biological active agent.

In this study the synthesis and characterization of collagen/hydroxyapatite drug delivery systems (COLL/HA/active agent - COLL/HA-AAg) is presented. Four active components were used: acetylsalicylic acid, salicylic acid, acetaminophen and niflumic acid in order to use them in pain or inflammation management. The purpose of the use of these active agents is derived from the high risk of inflammation and pain that occurs upon the surgical intervention.

Hydroxyapatite was obtained by precipitation using $\mathrm{Ca}(\mathrm{OH})_{2}$ and $\mathrm{NaH}_{2} \mathrm{PO}_{4}$ as precursors. By short, the COLL/HA composite material was obtained by the two steps mineralization process [1]. In the first step, calcium hydroxide is added to the collagen gel and allowed to interact for $24 \mathrm{~h}$. In the second step, the sodium dihydrogen phosphate solution is added to the above presented reaction mass leading to the precipitation of hydroxyapatite. The mineralization process was carried out at a precipitation $\mathrm{pH}$ of $9.5-10$ (dilute $\mathrm{NaOH}$ or $\mathrm{HCl}$ solution is used accordingly to maintain the $\mathrm{pH}$ ) and a temperature of $35 \pm 2{ }^{\circ} \mathrm{C}$. In order to obtain COLL/HA/AAg drug delivery systems, the composite material obtained by mineralization of the collagen gel is loaded with the appropriate amount of active agent followed by cross-linking with glutaraldehyde. Finally, the obtained composite materials and corresponding drug delivery systems were dried by freeze-drying.

In order to determine the structure and morphology of the multifunctional materials, the COLL/HA/AAg drug delivery systems were investigated by Fourier transform infrared spectroscopy (FTIR), X-ray diffraction (XRD) and scanning electron microscopy (SEM). The delivery profile of the active agents was studied by monitoring the conductivity of the solution as well as by UV-VIS spectroscopy and are essential in the pain and inflammation management after the surgical intervention associated with the grafting. All the delivery studies were carried out in PBS (phosphate buffer saline solution at $37 \pm 1^{\circ} \mathrm{C}$.

Keywords: collagen, hydroxyapatite, drug delivery system

\section{Acknowledgements}

The present work was possible due to the EU-funding grant POSCCE-A2O2.2.1-2013-1, Project No. 638/12.03.2014, code SMIS-CSNR 48652. The financial contribution received from the national project "Structuri poroase biomimetice obtinute prin printare 3D pentru ingineria tesutului osos (Acronim: BIOGRAFTPRINT), Nr 127PED/2017 is also highly acknowledged. 


\section{References}

[1] A. Ficai, E. Andronescu, G. Voicu, C. Ghitulica, B. S. Vasile, D. Ficai, V. Trandafir, "Self-assembled collagen/hydroxyapatite composite materials," Chemical Engineering Journal, vol. 160, pp. 794-800, 2010.

[2] A. Oryan, S. Alidadi, A. Moshiri, N. Maffulli, "Bone regenerative medicine: classic options, novel strategies, and future directions," Journal of Orthopedic Surgery and Research; vol. 9, no. 1, p. 18 (doi: 10.1186/1749-799X-9-18), 2014. 